\title{
Optimización de costos de inventarios con algoritmo de programación lineal. Caso aplicado industria de producción de suelas
}

\section{Cost Optimization of inventory with lineal program algorithm. Case applied in shoe soles industry production}

\author{
Darwin Aldás Salazar \\ John Reyes Vásquez \\ Luis Morales Perrazo \\ Santiago Sánchez Sánchez \\ Universidad Técnica de Ambato, Ecuador
}

Autor para correspondencia: darwinsaldas@uta.edu.ec

Fecha de recepción: 15 de diciembre 2017 - Fecha de aceptación: 26 de febrero de 2018

Resumen: En la presente investigación se plantea un algoritmo matemático de programación lineal que evalúa las condiciones que intervienen en los inventarios de materias primas almacenados en una industria de producción de suelas para zapatos tales como la demanda, el límite de la capacidad productiva, y los costos relacionados por llevar inventario; se genera una función objetivo y las respectivas restricciones del sistema, el mismo que se modela y resuelve en un software de programación Lingo®, el algoritmo permite la optimización de las cantidades de material a requerir, inventario al final de cada período y la cantidad de unidades faltantes con la finalidad de satisfacer la demanda y al mismo tiempo minimizar el costo total del inventario maximizando las utilidades en un horizonte de planeación de doce meses. Como resultado el modelo matemático planteado para el control de inventario de materia prima genera un ahorro de dinero anuales de $\$ 52566,50$ que corresponde a una mejora del $25.6 \%$ respecto al modelo actual.

Palabras clave: algoritmos; optimización; control de inventarios; programación lineal; costos

Abstract: In this research it has been proposed a mathematical algorithm of lineal programming that evaluates the conditions that intervene in the inventories of raw materials stored in a shoe soles industry such as the demand, the limit of the productive capacity, and the related costs of carrying inventory; it is generated an objective function and its respective restrictions of the system, the same that is modeled and solved in a Lingo® programming software, the algorithm enables the optimization of the amounts of material to be required, inventory at the end of each period and the amount of missing units with the finality of satisfying the demand and both minimizing the total cost of the inventory maximizing the profit in planning's horizon of twelve months. As a result, the mathematical model presented to the control of raw material inventory that generates saving money per year of $\$ 52566.50$, which come to an improvement of $25.6 \%$ with respect to the current model.

Key words: algorithms; optimization; inventory control; linear programming; costs 


\section{Introducción}

Las industrias de producción para la fabricación de sus productos adquieren materiales e insumos que se almacenan en inventarios de bodega, algunas veces por tiempos prolongados generando así costos innecesarios para la empresa.

Los inventarios, definidos de manera general, son aquellos artículos a la mano destinados para consumo. El control de inventarios está integrado por técnicas para determinar cuándo deben reabastecerse los inventarios actuales y cuánto debe reabastecerse. (Solow \& Mathur, 1996)

La aplicación de un Sistema de Gestión de Inventarios es una de las alternativas más influyentes en el esfuerzo por reducir los costos y mejorar la eficiencia económica, en este contexto el análisis cuantitativo de los problemas de inventario ha probado ser una herramienta importante para la toma de decisiones acerca de las variables principales que definen el comportamiento y costo de los inventarios. (Diaz Batista \& Pérez, 2013).

Tungurahua es una provincia en la cual se han desarrollado en gran cantidad industrias manufactureras de calzado y a su alrededor fábricas de materias primas e insumos tales como cuero o suelas. Lilia Villavicencio presidenta de la Cámara de Calzado de Tungurahua (CALTU), menciona que en la provincia se fabrica el $50 \%$ de la producción de zapatos en el Ecuador. (MIPRO, 2015)

Una herramienta para la optimización de operaciones es la programación lineal, ya que es de gran ayuda para la toma de decisiones, puesto que se ajustan a la realidad del problema y brindan soluciones óptimas de acuerdo al objetivo planteado, permitiendo minimizar o maximizar el mismo. (Caicedo \& Ortiz , 2014)

El objetivo de la presente investigación es utilizar un algoritmo de programación lineal que permita conocer las cantidades óptimas de requerimiento de materias primas con el fin de no tener almacenada en bodega y evitar costos innecesarios.

\section{Fundamentos teóricos}

Un eficiente control de inventarios trata de mantener un nivel de stock y permite, garantizar el flujo de material con la calidad requerida, en el lugar y momento oportuno y a un mínimo de costo, y así lograr un máximo de servicio a los clientes, es importante gestionar los inventarios dentro de la logística empresarial. (Parada Gutierrez, 2009)

Modelos específicos de la investigación de operaciones como la programación lineal son de gran ayuda para la toma de decisiones dentro de la empresa, con este enfoque investigaciones utilizan modelos determinísticos con base a datos reales, logrando un resultado óptimo que sugiere cuánto producir, cuánto demorar y cuánto almacenar en un horizonte de tiempo, minimizando los costos para un producto en especial (Ospina Gutierrez \& Rodas Rendón, 2008). 
Se consideran los costos de inventarios en la función objetivo de los modelos matemáticos, como una aproximación proporcional a la demanda promedio (Fernández Guédez, 2011).

Un programa óptimo de producción, constituye una herramienta con la cual el empresario puede generar una ventaja frente a la competencia y ser más productivo, mientras maximiza sus utilidades o throughput. (Caicedo \& Ortiz, 2014)

De entre varias herramientas de optimización, Lingo® es utilizada para resolver problemas de programación lineal, este software posee algoritmos que facilitan solucionar por ejemplo un plan de requerimiento de materiales (Caseres \& Reyes, 2014).

\section{Métodos}

Para el desarrollo de la investigación se planteó un modelo para el control de inventario de materia prima considerando el problema de programación lineal planteado por Ponsot y Márquez, con el fin de obtener científicamente una política óptima para el manejo de inventario de materia prima, que considera variables de estudio tales como: costos relacionados con los inventarios, la demanda, la capacidad de producción, el inventario inicial (Ponsot \& Marquez, 2000). Las variables de las restricciones se muestran en la tabla 1.

Tabla1: Modelo de programación lineal par inventario (Ponsot \& Marquez, 2000)

\begin{tabular}{ll}
\hline Variable & \multicolumn{1}{c}{ Descripción } \\
\hline & Número de períodos: cantidad de períodos en que se divide el horizonte de planeación para el cual se \\
$\mathrm{k}$ & ejecuta el modelo, en este modelo $\mathrm{k}=12$. \\
$\mathrm{xi}$ & Número de unidades producidas en el i-ésimo período, $\mathrm{i}=1, \ldots, \mathrm{k}$. \\
$\mathrm{yi}$ & Número de unidades en inventario al final del i-ésimo período, $\mathrm{i}=1, \ldots, \mathrm{k}$. \\
$\mathrm{zi}$ & Número de unidades no satisfechas en el i-ésimo período, $\mathrm{i}=1, \ldots, \mathrm{k}$. \\
& \\
$\mathrm{di}$ & Número de unidades demandadas del producto en el i-ésimo período, $\mathrm{i}=1, \ldots, \mathrm{k}$. \\
yo & Nivel del inventario inicial del producto (al inicio del período 1 ). \\
& Límite de la capacidad productiva. Número máximo de unidades del producto que pueden ser \\
$\mathrm{L}$ & producidas en un período cualquiera. \\
$\mathrm{C} 1$ & Costo unitario del producto. \\
$\mathrm{C} 2$ & Costo unitario de mantener. \\
$\mathrm{C} 3$ & Costo unitario de escasez.
\end{tabular}

La función objetivo con la que se logra optimizar el inventario de materias primas se muestra en la ecuación (1):

$$
C=C_{1} \sum_{i=1}^{k} X_{i}+C_{2} \sum_{i=1}^{k} Y_{i}+C_{3} \sum_{i=1}^{k} Z_{i}
$$


Población y Muestra: la empresa para su producción de suelas dispone de alrededor de 68 artículos en su bodega de materia prima, por lo que para el presente estudio se realiza un análisis ABC de los artículos con la finalidad de obtener los de mayor demanda, siendo estos 8 (Isocianato duro 99; Poliol suave 123; Isocianato suave 03; Poliol duro 350; Percloretileno; Catalizador duro; Cloruro de metileno; Pasta epaflex negro)

Análisis de los datos: Una vez definido el modelo de programación lineal se procede a aplicar el mismo para los ocho artículos cruciales definidos en el estudio ABC, posteriormente se resuelve los modelos planteados mediante el uso de la herramienta de optimización Lingo®, diseñada para hacer de la elaboración y la solución de modelos de optimización más rápido, más fácil y más eficiente.

Para la programación de los modelo de programación lineal en Lingo®, se consideran los comandos, operadores y funciones utilizados para resolver el modelo matemático.

\section{Resultados}

\section{Proceso de producción de suelas hechas en poliuretano}

Para la realización del producto, se utiliza un proceso conocido como inyección directa al corte, en la que dos materiales básicos, el poliol e isocianato en estado líquido se mezclan y forman el poliuretano. En su mayoría las suelas se producen bajo pedido y en ciertas ocasiones se cuenta con un inventario mínimo que permite cubrir pequeños pedidos. Se debe resaltar que el proceso de producción es el mismo para las todas las suelas sin importar la talla o la población a quien va dirigido.

\section{Análisis del inventario}

En bodega existen dos grupos de inventario: el inventario de materia prima y el inventario de producto terminado, siendo la materia prima la predominante en cantidad. Actualmente la empresa no cuenta con una política bien definida para el manejo de inventario, puesto que el mismo está en función de la demanda y ésta en cuanto a los pedidos de los clientes. Por lo que es difícil conocer cuándo se debe realizar un pedido al proveedor y en qué cantidad, al mismo tiempo no se cuenta con un registro exacto de los stocks.

De cada uno de los materiales estudiados se determinó la cantidad que se pide cada año y el costo total.Por ejemplo de isocianato duro99 actualmente según el estudio se ha pedido en un año $21840 \mathrm{Kg}$, y según el modelo de programación lineal y considerando las variables de estudio como la demanda, el límite de la capacidad productiva, los costos unitarios del artículo, costos de mantenimiento y costo por faltantes para cada mes del horizonte de planeación, se requiere $18494,33 \mathrm{Kg}$ con un costo mínimo de $\$ 81920,43$, si lo comparamos con el costo del producto actualmente más lo que representa mantenerlo en bodega es de 89978,40 logrando un ahorro del $8,96 \%$. 
Es importante recalcar que los resultados modelados en el programa se lo realizó para cada mes en un horizonte de planeación de 12 meses , permitiendo así verificar en cada mes si la cantidad abastecida en cada período satisface la demanda para la producción requerida.

\section{Análisis de resultados}

El modelo matemático planteado para el control de inventario de materia prima de los demás materiales se resume en la Tabla 2, y se determina el ahorro de dinero que proporciona el mismo, el porcentaje de mejora y el promedio de los porcentajes en cada material

Tabla 2: Resultados del estudio

\begin{tabular}{|c|c|c|c|c|c|}
\hline Descripción & $\begin{array}{l}\text { Cantidad a pedir } \\
\text { (Kg/año) }\end{array}$ & $\begin{array}{l}\text { Costo } \\
\text { Actual } \\
\end{array}$ & $\begin{array}{l}\text { Costo } \\
\text { Optimo }\end{array}$ & $\begin{array}{l}\text { Ahorro de dinero } \\
(\$ / a n ̃ o)\end{array}$ & $\begin{array}{l}\% \\
\text { Mejora }\end{array}$ \\
\hline Isocianato duro 99 & 18494,33 & 89978,4 & 81920,43 & 8057,97 & 8,96 \\
\hline Poliol suave 123 & 10948,61 & 67246,5 & 45441,75 & 21804,75 & 32,41 \\
\hline $\begin{array}{l}\text { Isocianato suave } \\
03\end{array}$ & 12282,38 & 62590,43 & 50509,98 & 12080,45 & 19,3 \\
\hline Poliol duro 350 & 10733,36 & 51172,2 & 47593,12 & 3579,08 & 6,99 \\
\hline Percloretileno & 3206,59 & 7612,18 & 5251,61 & 2360,57 & 31,61 \\
\hline $\begin{array}{l}\text { Catalizador duro } \\
\text { Cloruro de }\end{array}$ & 2267,59 & 10243,44 & 10046,96 & 187,48 & 1,83 \\
\hline metileno & 4050,27 & 7380 & 3794,45 & 3585,55 & 48,58 \\
\hline Pasta negro & 164,18 & 1650 & 739,35 & 910,65 & 55,19 \\
\hline Total & & & & 52566,5 & 25,6 \\
\hline
\end{tabular}

\section{Conclusiones}

Con el modelo matemático propuesto para el control de inventario de materia prima se obtiene las cantidades específicas a ser requeridas en base a la minimización del costo total de inventario. Un ejemplo particular para el material isocianato duro 99 indica que se debe pedir la cantidad anual de $18494.33 \mathrm{Kg}$, lo que refleja una disminución del costo total por manejo de inventario, representando un ahorro de 8057.97 dólares equivalente a una mejora del 8,96 \% en relación al modelo de control de inventario que se venía aplicando en la empresa, en promedio con los demás artículos se logra un ahorro de dinero anuales de \$52566,50 que corresponde a una mejora del $25.6 \%$ respecto al modelo actual.

Para la empresa el estudio le sirve de gran apoyo, ya que un mejor control de inventario brinda no sólo soluciones al problema de costo, a la vez permite que la empresa considere un cambio en las políticas y toma de decisiones en cuanto a productividad, manejo del flujo total de materiales y desenvolvimiento del mismo, permitiendo optimizar sus resultados de manera que se obtenga el mayor beneficio.

\section{Agradecimientos}


La presente investigación se lo realiza como aporte al proyecto de investigación DIDE de la Universidad Técnica de Ambato titulado "Optimización operacional basada en un sistema dinámico esbelto de alerta de fallas en los procesos de producción para las industrias de calzado" coordinado por el Ing. Mg. John Reyes, por lo cual se agradece su apertura para aportar con trabajos investigativos en dicho proyecto.

\section{Bibliografía}

Caicedo, Á. J., \& Ortiz, V. K. (2014). Procedimiento para la programación y control de la producción de una pequeña empresa de calzado. Scientia et Technica, 19(4).

Caseres, D., \& Reyes, J. (2014). Modelo de programación lineal para planeación de requerimientos de material en "Carrocería M\&L. Ambato.

Chase, R., Jacobs, R., \& Aquilano, N. (2009). Administración de operaciones, producción y cadena de suminitros. Mexico: Mc Graw Hill.

Devoto Ratto, R., \& Ruiz Vidal, E. (2003). Programación lineal para administración. Valparaíso: Ediciones universitarias de Valparaíso, Pontificia Universidad Católica de Valparaíso.

Diaz Batista, J. A., \& Pérez, D. (2013). Optimización de los niveles de inventario en una cadena de suministro. Ingeniería Industrial, 130-132.

Díaz Fernández, B., \& Del Brio González. (2001). Modelización de un DSS para la gestión de productos perecedores. Qüestiió, 25(2).

Fernández Guédez, C. (2011). Programación lineal e ingeniería industrial: una aproximación al estado del arte. Ingeniería Industrial.

MIPRO. (2015).

Mipro. (02 de 07 de 2015). Ministerio de Industrias y Productividad. Recuperado el 11 de 10 de 2015, de http://www.industrias.gob.ec/bp131-ficce-2015-oportunidad-para-conocer-a-laindustria-del-calzado-ecuatoriano-con-calidad/

Ospina Gutierrez, L. M., \& Rodas Rendón, P. A. (Diciembre de 2008). Modelo de programación para integrar producción, inventario y ventas en empresas industriales. Scientia et Technica, 1(40).

Parada Gutierrez, O. (2009). "Un enfoque multicriterio para la toma de decisiones en la gestión de inventarios," Competitividad, eficiencia y calidad en la gestión empresarial: entorno cultural y cultura organizacional. Proyecto de investigación de la Facultad de Ciencias Económicas y Empresariales de la Universidad de Oriente, 1.

Ponsot, E., \& Marquez, V. (2000). Modelo de programación lineal de la prodcucción, integrado en un sistema computarizado de producción, inventario y ventas industrial. Economía. 
Reyes, J., \& Molina, C. (2014). Plan agregado de producción mediante el uso de un algoritmo de programación lineal: un caso de estudio para la pequeña industria. Revista politécnica.

Solow, D., \& Mathur, K. (1996). Investigación de operaciones. México D.F., México: PrenticeHall. 